\title{
L’interface disciplinaire comme révélateur des liens environnement-santé
}

\author{
G. Maignant \\ ESPACE, Université de Nice Sophia-Antipolis, 06204 Nice Cedex, France
}

\begin{abstract}
Résumé. Comprendre les liens fonctionnels et causaux entre l'environnement et la survenue de certaines pathologies est devenu un enjeu de société. Cet enjeu ne peut se concevoir au cœur d'une discipline spécifique, mais c'est bien à l'interface entre les sciences dites «exactes》 et les «sciences humaines et sociales》 que le débat s'invite. Cette double interface disciplinaire et thématique permet d'appréhender l'acceptation sociale de mesures environnementales proposées, suite à des modélisations spécifiques. Issu, d'un double cursus universitaire de mathématiques appliquées et de géographie, l'auteur de cette contribution analysera la question des seuils à travers des modèles comme la percolation (propagation d'un phénomène dans l'espace selon une direction privilégiée) ou encore la logique floue (argumentaire de la pertinence des seuils). Cette analyse sur le seuil, qui se veut à la fois quantitative et philosophique (le seuil comme élément de rupture dans l'événement spatial) questionnera l'optimalité des liens environnementsanté à différentes échelles spatiales et temporelles. Ainsi, nous souhaitons développer dans cette communication comment des liens invisibles entre urbanité, problèmes environnementaux et santé, peuvent être révélés dans une dynamique pluridisciplinaire complexe et complémentaire. Cette contribution qui se veut, avant tout, théorique s'appuiera néanmoins sur des exemples concrets illustrant comment une approche pluridisciplinaire peut être considérée comme révélateur de liens nouveaux entre environnement et santé. La question de la résilience confrontée à l'optimalité notamment en termes d'adaptabilité sera introduite et une discussion sera lancée sur comment faire de la prospective aujourd'hui ou encore demain ? Quels sont les déterminants de l'existant mobilisables pour d'une part mieux comprendre les liens environnement-santé ; d'autre part réduire la part de l'environnement dans la prévalence de certaines pathologies, notamment respiratoires.
\end{abstract}

\section{L'évolution des sciences ou l'interface disciplinaire...}

Les sciences ont beaucoup évolué lors de la dernière décennie, notamment grâce à la puissance des supercalculateurs mais aussi par la création de nouveaux concepts transdisciplinaires. En effet, les approches pluridisciplinaire et transdisciplinaire se sont récemment imposées comme incontournables dans la compréhension de phénomènes de plus en plus complexes pour lesquels aucune science, aussi puissante soit elle, ne pouvait apporter de solutions satisfaisantes. Bien qu'intellectuellement stimulante, cette approche à l'interface de disciplines bien sectorisées, n'est pas aussi évidente à instaurer. Tout le monde parle d'interdisciplinarité, en prône son intérêt mais pourtant la recherche continue à être segmentée à l'exception de rares contre-exemples ; citons notamment les sections CNRS ou encore plus les sections CNU qui restent des exemples de découpages scientifiques parcellaires peu fructueux à la mutation de concepts entre disciplines, de même que l'enseignement dans les classes du secondaire. Audelà de ce frein culturel et historique, cette difficulté première vient du fait que les scientifiques ont tous un langage qui leur est propre qui constitue la première frontière (interface scientifique) à la connaissance de l'existant dans d'autres sciences, mais aussi de la société civile, tant sur l'aspect thématique, que sur l'aspect méthodologique. En effet, celle-ci est peu préparée à recevoir un message scientifique non vulgarisé, voir même simplifié à outrance. Quelques exemples de transferts réussis des sciences mathématiques ou de l'ingénieur vers les sciences humaines et sociales peuvent toutefois être mentionnés. Tout d'abord la théorie fractale avec les travaux d'André Dauphiné (Dauphiné, 1995) ou de Pierre Fankhauser (Fankhauser, 1993) appliqués à la géographie urbaine et à l'étude des réseaux, la théorie constructale appliquée à travers une «géographie du mieux» à la qualité de vie, à la mixité sociale et à l'aménagement durable optimal (Maignant, 2009), ou encore la théorie de la relativité d'échelle, servant à analyser les rapports multi-scalaires qu'entretiennent les processus, très utiles en géographie aussi bien humaine que physique (Forriez, 2010, Martin, 2007). Ces transferts de théories mathématiques ou physiques ont beaucoup apporté à la géographie humaine et physique. Toutes les théories utilisées en géographie ou aménagement du territoire proviennent d'autres disciplines, essentiellement des sciences dites «dures ». Denise Pumain souligne d'ailleurs que «la mathématisation du champ n'est qu'un élément dans une vaste entreprise collective de reconstruction de la géographie » (Pumain, 2002). Pour Edgar Morin, «la science serait totalement embouteillée si les concepts ne migraient pas clandestinement. Mandelbrot disait que les grandes découvertes sont le fruit d'erreurs dans le transfert des concepts d'un champ à un autre, opérées, ajoutait-il, par le chercheur de talent» (Morin, 2005). Ainsi toutes les disciplines se nourrissent des transferts et des importations de leurs consœurs concurrentes ou complémentaires, puisque la science et les sciences sont des 
systèmes ouverts, en interaction et en perpétuel changement (Maignant, Emsellem, 2006). L'interdisciplinarité effective, permet alors de dépasser la simple juxtaposition de visions disciplinaires du même objet d'étude dans une approche complexe et globalisante; chaque science apportant ses théories, les confrontant aux autres dans cet aller-retour permanent entre modèles, théories et réalités. Bien entendu, il y a des thématiques ou des terrains d'étude qui se prêtent davantage à une approche à l'interface de plusieurs disciplines, c'est notamment le cas de la thématique environnement-santé, dont la multiplicité des facteurs et des approches reflètent à la fois la complexité de l'objet d'étude et son intérêt tant scientifique qu'à destination de la société civile. Nous questionnons maintenant la notion de seuil comme interface environnement-santé.

\section{La question des seuils - exemples de la percolation et de la logique floue}

Très présent dans les sciences humaines et sociales, notamment en économie, le seuil est souvent perçu comme étant une interface entre deux espaces bien différenciés (phénomène en tout ou rien) or au-delà de la quantification précise de ce seuil qui n'est déjà pas qu'une simple question mathématique, l'interprétation du phénomène considéré aux marges de cette interface est tout aussi délicate. Lena Sanders précise d'ailleurs que «seule l'éventuelle existence d'un effet de seuil [...] peut être interprétable dans la réalité géographique [car] il y a un réel problème d'identification de la valeur mathématique d'un paramètre avec sa signification géographique » (Sanders, 1992). Il est particulièrement délicat d'appréhender la question du seuil dans les théories en sciences humaines et sociales. Sans être exhaustif nous discutons dans cet article de deux théories utilisant la notion de seuil, l'une très déterministe (la théorie de la percolation correspondant à du tout ou rien), l'autre permettant d'approcher le seuil selon un espace imprécis et flou (la logique floue).

La percolation est un phénomène «en tout ou rien », elle fonctionne en fonction de seuil, dépendant de la forme du réseau considéré (mailles carrées, triangulaires ou autres). Pour illustrer cette notion de seuil de percolation, imaginons le cas d'un torrent qu'il faut traverser à pieds secs. Des rochers parsèment le cours d'eau. En parcourant l'une des deux rives, on trouve, enfin, un passage où les rochers sont suffisamment rapprochés pour permettre de passer à pieds secs d'une rive à l'autre. La densité de rochers apparents (sortant de l'eau) a atteint ce que l'on appelle le seuil de percolation. Un autre exemple simple est celui du lait, en supposant que vous vouliez le faire bouillir. Pour une température faible le lait chauffe mais, dès qu'il atteint une certaine température (température seuil), il se met à bouillir et à déborder (si vous n'êtes pas assez rapide pour enlever la casserole du feu). Le seuil correspond, scientifiquement parlant, au moment où l'amas percolant devient infini, c'està-dire qu'au-delà du seuil, il existe au moins un chemin voire plus qui permet de traverser le réseau de part en part. Le premier amas infini est appelé amas critique ${ }^{1}$. Ce type de transition de phase apparaît aussi dans les écrits de René

\footnotetext{
${ }^{1}$ En anglais, spanning cluster.
}

Thom, mathématicien français et inventeur de la théorie des catastrophes, en les termes : «par exemple, de l'eau que l'on fait bouillir contient au début de petites bulles de vapeur d'eau qui, initialement instables, finissent par grossir, s'agglomérer, et occuper tout le fluide » (Thom, 1991). Le moment où les petites bulles finissent par occuper tout le fluide correspond donc à l'amas percolant.

En matière d'environnement et de santé, la difficulté d'interpréter le seuil vient notamment de la complexité à exploiter la relation dose-réponse (espace flou) et de sa possibilité de validation sur des cas témoins. En effet, de mêmes doses ne produisent pas sur les individus les mêmes effets, ce qui signifie que cette interface doseréponse est non-linéaire et chaotique. Par exemple en matière d'immissions, c'est-à-dire de charge de pollution de l'air respirée (charge polluante présente dans l'air après dispersion en fonction de la morphologie urbaine et de la climatologie locale), il est très difficile de quantifier à partir de quelle quantité de polluants, on est susceptible d'avoir un effet sur la santé, d'autant plus que cela dépend également de la plus ou moins grande vulnérabilité du récepteur. L'état de santé du récepteur, dont le système immunitaire et/ou respiratoire peut être moins développé (enfants) ou fragilisé (femmes enceintes, fumeurs, personnes âgées ...) constitue, bien au-delà du seuil de polluant, l'un des facteurs discriminants majeurs. De plus, de mêmes doses de polluants respirées peuvent contribuer à des effets différents, selon leur combinaison chimique ; leur nocivité résultante n'étant pas la somme des nocivités individuelles. Ainsi, les valeurs guides ou limites, fixées par l'OMS ne sont que des valeurs de recommandations, représentant des normes politiques ou décisionnelles, elles ne seraient constituer une interface, entre deux espaces sains et pollués. Cependant, nous ne remettons pas en cause la mise en place de telles normes, elles sont une première approche nécessaire mais non suffisante.

Examinons maintenant, une autre méthode permettant de nuancer l'approche en «tout ou rien» de la percolation. Cette approche est la logique floue qui a été introduite par Zadeh en 1965, pour réduire les coûts de développements de logiciels. Ce concept de logique floue a été développé pour faire face à l'incapacité de classer des phénomènes en deux groupes : vrai ou faux (variables booléennes), la plupart des phénomènes réels devant être appréhendée par une variation progressive. La logique floue repose sur une modélisation des entrées d'un système par des courbes décrivant les degrés d'appartenance aux différents états identifiés des entrées. Les différents degrés d'appartenance se composent selon deux logiques : la logique «ou» et la logique «et». Dans le cadre de la logique «ou», on considère le degré d'appartenance maximum parmi les conditions d'entrée, tandis que dans le cadre de la logique «et» on considère le degré d'appartenance minimum (logique ensembliste). Le choix du degré d'appartenance maximum et minimum est similaire à celui de la théorie des intervalles. En effet, lorsque l'on cherche l'ensemble des éléments qui appartiennent à l'un ou l'autre des deux intervalles, cela signifie l'union des intervalles soit l'ensemble maximum (A est inclus dans l'union, B aussi), tandis que pour les éléments qui appartiennent à l'un et à l'autre des deux intervalles, cela signifie l'intersection et donc l'intervalle minimum ( $\mathrm{A} \cap \mathrm{B}$ est inclus dans $\mathrm{A}$ et 


\begin{tabular}{|c|c|c|}
\hline $\begin{array}{c}\text { Fonction } \\
\text { d'appartenance }\end{array}$ & $\begin{array}{c}\text { Logique } \\
\text { classique } \\
\text { (binaire) }\end{array}$ & $\begin{array}{c}\text { Logique } \\
\text { floue }\end{array}$ \\
\hline$\mu_{\mathrm{A}}(\mathrm{X})$ & 0 ou 1 & $\in[0,1]$ \\
\hline
\end{tabular}

Fig. 1. Fonction d'appartenance selon les deux logiques.

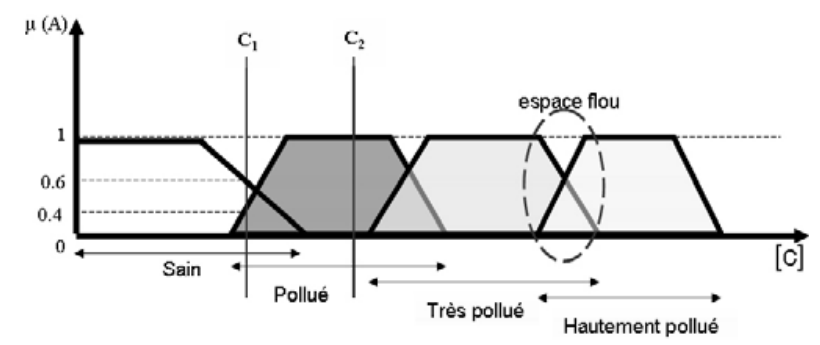

Fig. 2. Principe de la logique floue : modalités et fonction d'appartenance.

dans B). La logique floue est basée sur les ensembles flous (fonction d'appartenance ou probabilité d'appartenance à un ensemble permettant ainsi d'apporter toutes les nuances possibles), tandis que la logique classique (binaire) est basée sur la fonction d'appartenance $\left(\mu_{A}(\mathrm{X})\right)$ d'un ensemble classique autrement appelé le symbole de Kronecker.

La logique floue est particulièrement bien adaptée à l'appréhension de phénomènes pour lesquels une perception humaine peut être introduite, c'est notamment le cas des itinéraires urbains sous contraintes environnementales. En effet, le citadin, soucieux de sa santé, qui souhaite se rendre d'un endroit à un autre cherche ainsi à optimiser son parcours environnemental c'est-à-dire emprunter les axes les moins pollués possibles, le niveau de pollution de chaque axe variant de manière continue de 0 à $1(0$ signifiant un axe non pollué (sain), 1 , un axe irrespirable ${ }^{2}$ : hautement pollué). Bien entendu dans ce type d'analyse, on admet que l'usager a une bonne connaissance des niveaux de concentrations de polluants des axes, ce qui vraisemblablement sera bientôt une généralité, notamment grâce à la publication des cadastres d'émissions des villes et des panneaux d'information en temps réel qui tendent à se généraliser dans les grandes villes. Paris, dispose à ce jour de 170 panneaux lumineux à messages variables, implantés dans les rues, donnant une information en temps quasi réel sur la qualité de l'air ; mais aussi par le développement futur d'applications pour cellulaires (Android, iPhone).

Le concept de logique floue permet d'introduire des nuances dans la perception de la pollution des artères urbaines par le citadin (cartes mentales des espaces les plus pollués de la ville). En effet, elle est le seul moyen de traiter, dans un même cadre, des connaissances fournies numériquement par des instruments de mesure, et des connaissances exprimées symboliquement, par un observateur humain (Bouchon - Meunier, 1993). Si l'on prend l'exemple de la figure précédente, les différentes modalités (sain, pollué, très pollué, et hautement pollué) se recouvrent partiellement. Les zones de recouvrement sont appelées les espaces flous. Le flou ne signifie pas un manque de précision mais une diversité de perception. En effet, les modalités sont subjectives et reposent sur le présupposé

\footnotetext{
${ }^{2}$ Un axe irrespirable est défini comme un axe dépassant de manière notoire les seuils réglementaires.
}

de chacun. Des personnes particulièrement sensibles à la pollution (les plus vulnérables) auront tendance à fixer, à la baisse, la borne inférieure de chaque sous-ensemble. Si l'on prend une concentration $\mathrm{C}_{1}$ comme sur la figure précédente, l'entrée $\mathrm{C}_{1}$ appartient à $60 \%$ au sous ensemble « air sain », à $40 \%$ au sous ensemble «air pollué » et à $0 \%$ aux autres sous-ensembles. En revanche, la concentration $\mathrm{C}_{2}$ appartient intégralement au sous ensemble pollué. Ainsi, la logique floue permet de déterminer un domaine incertain autour d'une valeur seuil. Traditionnellement dans la logique floue, on évite que les modalités se recoupent plus de deux fois, ceci pour des raisons de stabilité mathématique et de programmation numérique, qu'il est inutile de détailler dans cet article. Le lecteur intéressé pourra se référer à l'ouvrage 《idées nettes sur la logique floue 》 de Godjevac (Godjevac, 1999).

La relation d'appartenance entre une variable (ici la concentration de polluants) et un sous ensemble de modalités : sain, pollué, très pollué, etc. se dit «fonction d'appartenance ». En d'autres termes, on parle de fonction d'appartenance d'une variable «a» à un sous-ensemble « $\mathrm{B} »$ et il est noté : $\mu_{\mathrm{B}}(\mathrm{a})$. Remarquons que la somme des fonctions d'appartenance n'est pas forcément égale à 1 , ce qui signifie notamment que les trapèzes de la figure précédente ne se coupent pas nécessairement à 0,5 . Notons également que la fonction d'appartenance choisie peut être différente (triangle ou autre) selon les besoins de modélisation plus ou moins complexe du phénomène.

La fonction d'appartenance doit cependant être continue. Ainsi toute valeur de l'intervalle $[0,1]$ est atteinte, mais elle peut être atteinte en plusieurs concentrations appartenant à des modalités différentes.

L'intérêt majeur de cette méthode est la prise en compte de la perception des populations (individuelle et collective), vécue ou ressentie (vulnérabilité de chacun, cartes mentales). Selon les pratiques quotidiennes ou historiques, chacun a une idée, plus ou moins précise, de la pollution de l'air à l'échelle urbaine (cartes mentales issues de pratiques antérieures et d'informations remises à jour, panneaux d'information, applications pour téléphonie mobile). Mais l'inconvénient de cette méthode est qu'elle nécessite des enquêtes de terrain pour fixer les différents seuils (concentrations et tolérance) et les degrés d'appartenance à ces différents seuils. Cependant, d'un point de vue purement conceptuel, elle apporte un enrichissement à la compréhension de l'interface environnement-territoire.

Pour une même concentration de polluants (par exemple $\mathrm{C}_{1}$ ), l'espace peut être perçu comme sain ou pollué, c'està-dire l'appartenance simultanée à deux sous-ensembles constituant l'interface qu'il est nécessaire de questionner en termes d'optimalité. Le seuil n'est pas déterminé de manière déterministe, comme dans la théorie de la percolation, mais correspond à une interface (zone de recouvrement). Dans la thématique environnement-santé qui nous intéresse, et selon la perception individuelle de l'homme, en fonction de sa propre vulnérabilité, une rue pourra être affectée de seuils de perception différents (filtres), constituant ces espaces flous, intégrant une vulnérabilité différenciée des individus.

Un optimum est toujours relatif à un contexte, aux contraintes afférentes et dépend de l'objectif que l'on se fixe (optimisation sous contraintes). Mathématiquement, 

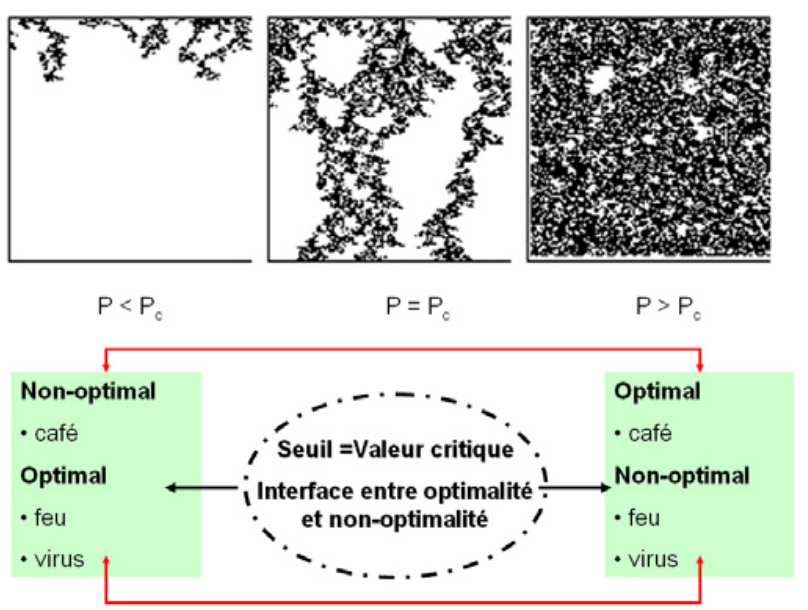

Fig. 3. Optimalité, le seuil comme valeur critique.

l'optimisation peut être comprise comme la maximisation ou la minimisation d'une fonction à plusieurs variables. D'un point de vue plus philosophique, l'optimalité n'est pas toujours la situation à rechercher comme on le verra par la suite.

Dans le cadre de la percolation d'un phénomène, le seuil est une valeur critique, constituant une interface entre deux situations distinctes : optimalité et nonoptimalité (Figure 3). Ainsi, selon le phénomène étudié, un positionnement clair vis-à-vis de la percolation optimale $\left(\mathrm{P}=\mathrm{P}_{C}\right)$ est à envisager (percolation sous-optimale dans le cas d'un feu de forêt ou d'une épidémie, percolation suroptimale dans le cas du café...).

Questionner le seuil comme interface permet de se repositionner dans la chaîne sous-optimale, optimale, sur-optimale, en invitant l'homme à se décaler parfois de l'optimalité et rappelle d'une part que l'optimum n'est pas toujours souhaitable (possibilité d'augmenter les facteurs de risques), d'autre part que l'optimum est toujours contraint par un système de valeurs et une finalité (optimum pour qui ?, optimum pour quoi ?, optimum comment ?). Le positionnement autour d'une valeur seuil est partie intégrante de l'analyse post-optimale permettant de repenser la prospective, non plus comme un seul ensemble de scénarios sur l'avenir, mais comme un recadrage de l'existant. Par exemple, dans le cadre d'un feu de forêt, les services d'incendie essayent de refaire passer la densité d'arbres en feux du côté inférieur au seuil de percolation (optimalité, dans ce cas) car sinon ils savent, par expérience, que le processus risque de rentrer dans une phase d'irréversibilité, c'est-à-dire la propagation du feu à l'ensemble de la forêt (percolation totale et non-optimale). La recherche de cet optimum passe donc par la détection d'un seuil que nous appellerons seuil d'irréversibilité. Pour Beat Bürgenmeier, «l'analyse des seuils d'irréversibilité, tant dans la sphère sociale que dans la sphère environnementale, devrait servir à articuler les politiques de développement durable » (Bürgenmeier, 2004). Ainsi, l'aménagement du territoire devrait se faire sur la base de l'existant d'une part, sur la base d'éléments résilients d'autre part (nécessité de considérer les seuils d'irréversibilité) et sur un recadrage optimal de l'existant.

L'exemple du café est semblable à celui du feu mais l'optimalité est atteinte après le franchissement du seuil de percolation. En effet, lorsque l'on souhaite préparer un bon café, il est nécessaire de posséder un filtre à café optimal, c'est-à-dire que ses pores ne doivent être ni trop fins (sinon on attend le café un temps inacceptable), ni trop larges ( sinon le café est «light»). La percolation optimale se fait en ayant le bon filtre et le franchissement du seuil $\left(\mathrm{P}>\mathrm{P}_{C}\right)$. Même si la quantification du seuil reste encore une question ouverte ; le seuil constitue une interface optimal / non optimal.

\section{Résilience, optimalité et adaptabilité ou comment faire de la nouvelle prospective}

La question des interfaces, mise en relation avec la thématique environnement - santé, oblige le chercheur à questionner la pertinence de valeurs seuils comme nous l'avons vu mais également à se positionner face à une certaine forme d'adaptabilité des espaces, afin de leur proposer un avenir durable et optimal. Pour Olivier Labussière, «optimiser l'espace géographique peut être entendu selon une relation de cause à effet : l'optimisation comme interventions sur les conditions initiales d'un état du monde de façon à ce que leurs modifications génèrent un bénéfice attendu» (Labussière, 2008). Ainsi, cette vision proactive dépasse le caractère utopique d'un optimum inatteignable en prenant en compte, d'égal à égal, la recherche d'un bon existant et la construction d'un devenir souhaitable des territoires.

L'un des grands défis du $21^{\text {ième }}$ siècle est, à nos yeux, de savoir comment optimiser l'espace sur terre pour rendre suffisamment durable, notre mode d'habiter. La thématique environnement - santé est ainsi au cœur de cette réflexion pluri et transdisciplinaire. Le caractère opérationnel de ce questionnement intellectuel ne doit en aucun cas masquer la complexité sous-jacente de la question. L'optimisation, à travers la géographie du mieux, doit donc apporter son côté opérationnel à la construction d'un monde plus durable. Elle doit être perçue comme une clé de relecture du monde. La notion de «mieux» entraine implicitement le choix d'une «non-violence» par rapport à ce qui existe. Ce qui signifie, notamment qu'en matière d'aménagement, il ne s'agit pas de faire table rase du passé mais bien au contraire de faire «bouger» les éléments entre eux pour obtenir une nouvelle figure dont on pressent qu'elle est meilleure, au regard de critères complexes combinés. Ce mode de fonctionnement (recadrage de l'existant) est relayé par des conduites moins intrusives dans l'action et constitue un nouveau regard sur la prospective territoriale. Ce mode de fonctionnement, ce recadrage étape par étape est très proche de la méthode développée par le psychologue Paul Watzlawick, dont Jean Paul Ferrier rappelle les fondements « la méthode du recadrage consiste à modifier le contexte conceptuel et/ou émotionnel d'une situation, ou le point de vue selon lequel elle est vécue, en la plaçant dans un autre cadre, qui correspond aussi bien, ou même mieux, aux faits de cette situation concrète, dont le sens, par conséquent change complètement. [...] Ce qu'on modifie en recadrant, c'est le sens accordé à la situation, pas ses éléments concrets 》(Ferrier, 2000).

Un des autres aspects de la prospective qui mérite d'être souligné est qu'un ensemble de scénarios est habituellement 
donné sans quantifier le degré de croyance dans chacun des futurs proposés (Ha-Duong, 2005), et c'est une grossière erreur de vouloir y coller des probabilités subjectives à dire d'experts (méthodes bayésiennes) car comme le précise le célèbre prospectiviste, Peter $\mathrm{Wack}^{3}$, «I have a strong feeling that it will be poisonous and will contaminate the logic of scenarios». Rappelons qu'une situation la plus probable (au sens mathématique du terme) n'est pas forcément celle qui va se réaliser ou encore moins celle qui serait souhaitable, «le seul certain est le certain dont il est certain qu'il ne se réalisera pas » (Chamussy, 1998). Ainsi, Michel Godet souligne que «la probabilisation ne doit pas conduire à écarter de la réflexion des scénarios très peu probables, mais néanmoins importants en raison des risques de rupture et des impacts majeurs qu'ils signifient » (Godet, 2000).

\section{Conclusion}

Analyser les relations environnement-santé passe par une réflexion plus approfondie de la relation dose-réponse, autrement dit de la question des seuils. De même, la mutation de théories et méthodes entre disciplines devrait être plus encouragée, bien au-delà des discours prônant l'interdisciplinarité car « aucune science ne dispose d'objets propres, chaque discipline donne un point de vue propre sur des objets que d'autres disciplines peuvent envisager » (Charre, 2003). En effet, «la complexité est invisible dans des disciplines qui fragmentent l'objet ou qui l'isolent. D'où la nécessité de relier. Car dés que vous avez un objet riche multidimensionnel, il ne suffira pas d'additionner les disciplines.» (Morin, 2002). De par, les relations complexes, non-linéaires, que la santé et l'environnement entretiennent, seul le regard pluridisciplinaire permet d'apporter un nouveau souffle dans la compréhension de ces interfaces multidimensionnelles. La question de l'optimalité, quant à elle, est au cœur de cette réflexion. En effet, il ne s'agit plus seulement d'enrayer les nuisances environnementales pouvant agir sur la santé mais bien de proposer un devenir durable et souhaitable de nos territoires. C'est-à-dire un territoire optimisé, s'intégrant dans une 《géographie du mieux » (Maignant, 2009).

\section{Références}

1. A. Dauphiné, Chaos, fractales et dynamiques en géographie, Coll. Espaces modes d'emploi, Vol. 4 de Espaces modes d'emploi, Reclus, 135 p. (1995)

2. P. Frankhauser, La fractalité des structures urbaines, Economica, 462 p. (1993)

3. G. Maignant, Réflexion sur l'optimisation en géographie ou comment penser la «géographie du mieux», mémoire d'Habilitation à Diriger des Recherches, Université de Nice Sophia-Antipolis, 149 p. (2009)

\footnotetext{
${ }^{3}$ Peter Wack, cité par Ha Duong (2005, p.126)
}

4. M. Forriez, Caractérisation formelle des structures multi-échelles géographiques en relativité d'échelle, exemples en géographie physique, géographie urbaine, géohistoire et géographie du peuplement, thèse de Doctorat, Université d'Avignon et des Pays du Vaucluse, 408 p. (2010)

5. P. Martin, L. Nottale, «La théorie de la relativité d'échelle, une base commune à une vision structurelle du monde?», Textes pour la contribution aux débats du workshop, Université d'Avignon et UMR ESPACE 6012 du CNRS éditeur, seconde édition, 65 p. (2007)

6. D. Pumain et M.C. Robic, Le rôle des mathématiques dans une «révolution» théorique et quantitative : la géographie française depuis les années 1970, Revue des Sciences humaines, 2002/1, n6, pp. 123-144 (2002)

7. E. Morin, Introduction à la pensée complexe, Coll. Essais, Points Seuil, 158 p. (2005)

8. G. Maignant, K. Emsellem, «Géographie et mathématiques : quels liens existants, quelles potentialités ?», Actes du colloque Géopoint «Demain la géographie », Avignon, 1-2 juin, 8 p. (2006)

9. L. Sanders, Les modèles dynamiques : intérêts et limites en géographie, Géopoint 1992, pp. 159-163. (1992)

10. R. Thom, Prédire n'est pas expliquer, Coll. Champs, Flammarion, 174 p. (1991)

11. B. Bouchon-Meunier, La logique floue. Presses Universitaires de France, Coll. Que sais-je ?, 128 p. (1993)

12. J. Godjevac, Idées nettes sur la logique floue, Lausanne, Presses Polytechniques et Universitaires Romandes, coll. Informatique, 125 p. (1999)

13. B. Burgenmeïer, Economie du développement durable, Coll. Questions d'économie et de gestion. De Boeck, 272 p. (2004)

14. O. Labussiere, Optimisation, organisation de l'espace et pensée de l'émergence, la piste esthétique chez Gilles Deleuze, 8 p. (2008) http://hal. archivesouvertes.fr/docs/00/33/55/63/PDF/Labussiere_ Optimisation_organisation_de_l_espace_et_pensee_de_l_ emergence.pdf

15. J.P. Ferrier. Les très grandes villes dans le monde. Etude géographique, sous la direction de Jean-Pierre Paulet, Coll. CNED/SEDES, 48 p. (2000)

16. M. Ha Duong, Modèles de précaution en économie : introduction aux probabilités imprécises, Habilitation à diriger des recherches, Université Paris I - Panthéon Sorbonne, 165 p. (2005)

17. H. Chamussy, «Le certain, l'imprévu, l'obscur, le hasard aujourd'hui », Synthèse final du Géopoint 98, 8 p. (1998)

18. M. Godet, De la rigueur pour une indiscipline intellectuelle, in «La prospective stratégique d'entreprise» Lesourne (dir.), Dunod, 28 p. (2000)

19. J. Charre, «L'objet géographique, chose disciplinaire.» in MABY J. sous la direction de, Réflexion sur la nature des objets et des indicateurs de la géographie, pp. 308316. (2003)

20. E. Morin, «A propos de la complexité », Discours d'entrée CNRS, 14 janvier 2002. 